\title{
Flavor Physics at ATLAS: Status and Perspectives
}

\author{
Martin zur Nedden on behalf of the ATLAS Collaboration ${ }^{\text {a }}$ \\ ${ }^{a}$ Humboldt-University of Berlin, Institute of Physics, \\ Newtonstrasse 15, 12489 Berlin, Germany
}

In this note, the first flavor physics results of ATLAS based on an integrated luminosity up to $6.4 \mathrm{nb}^{-1} \mathrm{are}$ presented. This includes measurements of hidden and open charm production with $J / \psi$ and $D$-mesons. The agreement of the measured mass values with the PDG world averages and the signal widths with the Monte Carlo expectations is very good demonstrating a good understanding of the detector performance already with the first data. Furthermore, the tagging of $b$-jets based on decay length and impact parameters originating form $B$-meson decays is shown with first data. The note concludes with an outlook to the higher luminosity phase including aside from heavy quarkonia studies also the prospects to measure the rare $B$-decays such as as $B_{s} \rightarrow \mu^{+} \mu^{-}$and precise measurements for the $B$-meson family.

\section{The ATLAS Flavor Physics Program}

The ATLAS Experiment at the Large Hadron Collider LHC has a long-term flavor-physics program. Already within the first $10 \mathrm{nb}^{-1}$ of data, the well known standard model decays of $J / \psi$ and $\Upsilon$ mesons can be used for detector performance studies as tracking validation, alignment and trigger commissioning [1]. The anticipated integrated luminosity of $100 \mathrm{pb}^{-1}$ to be collected in 2010 , the first year of $p p$-collisions data taking, the measurement of production cross sections of $B$ hadrons, $J / \psi$ - and $\Upsilon$-mesons can be done to test the QCD predictions at LHC energies. In parallel, the performance studies will be continued by measuring the cross section ratios $b \bar{b} \rightarrow J / \psi X$ and $B^{+} \rightarrow J / \psi K^{+}$to $p p \rightarrow J / \psi X$. If up to the planned end of the first data taking phase by the end of $2011(\sqrt{s}=7 \mathrm{TeV})$ the total anticipated integrated luminosity of $1 \mathrm{fb}^{-1}$ will be reached, an extended study of the full $B$-meson family $\left(B^{+}\right.$, $\left.B_{s}, B_{c}\right)$ and $\Lambda_{b}$ will be possible. With the larger samples of the main $B$-decays to be collected with this data, contributions can be made to the measurements of the properties of $B$-mesons as well to set limits on rare decays ratios.

One of the most sensitive processes to new physics is the rare decay $B_{s} \rightarrow \mu^{+} \mu^{-}$. Since this process is heavily suppressed in the Standard
Model an integrated luminosity of $\approx 10 \mathrm{fb}^{-1}$ is needed, to reach the Standard Model (SM) limits. This will only be achieved in the data taking phase after the shutdown in 2012.

\section{First Measurements at $7 \mathrm{TeV}$}

A study of the $J / \psi \rightarrow \mu^{+} \mu^{-}$performance and $D^{* \pm}, D^{ \pm}$and $D_{s}^{ \pm}$reconstruction was done with the ATLAS detector in $p p$ collisions at $\sqrt{s}=$ $7 \mathrm{TeV}$ using the first data with an integrated luminosity up to $6.4 \mathrm{nb}^{-1}$. This was one of the first important steps to test and understand the performance and behavior of the ATLAS detector and trigger. A very good agreement of the reconstructed parameters with the world-averages PDG [2] values as well with the Monte Carlo (MC) expectations were found. The MC was generated with Pythia 6 [3], tuned using the ATLAS MC09 tune [4] and MRST LO* [5] parton distribution functions.

\subsection{Reconstruction of $J / \psi$ Mesons}

One of the goals of this study [6] was to understand the behavior of $J / \psi$ mesons at very low $p_{T}$ which can only be selected in the initial low luminosity phases of LHC. A loose selection is applied to understand additionally the shape of background. With respect to the trigger, only a minimum bias requirement is applied defined 
by an activity in the minimum bias scintillator (MBTS $2.9<|\eta|<3.89$ ) at both side of the detector. On the higher level trigger, additional activity in the muon system is required.

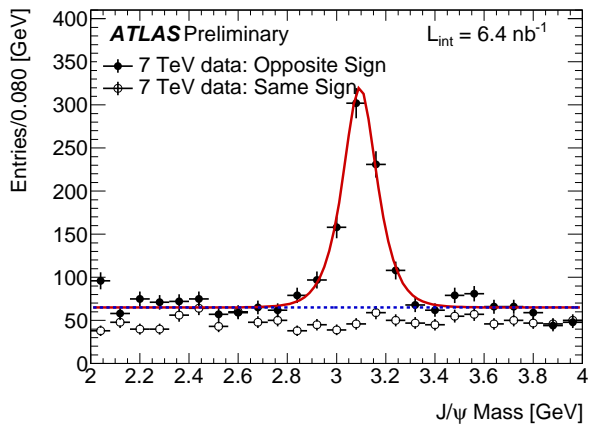

Figure 1. The invariant mass distribution for reconstructed $J / \psi \rightarrow \mu^{+} \mu^{-}$candidates from data (black points) after the selection with the same sign combinations (open points) superimposed. The solid line shows the result of a unbined maximum likelihood fit to all di-muon pairs in the mass range analyzed.

The requirements on the collision events are the presence of at least three tracks associated with the same primary vertex fulfilling a basic track quality applying the cut $p_{T}^{\text {track }}>0.5 \mathrm{GeV}$. For each muon, an additional inner detector track is required. In order for a muon to be associated in the muon system, a total momentum of at least $3 \mathrm{GeV}$ is necessary. A tagged muon is the combination of inner detector tracks extrapolated to the muon system. Within the mass region of the di-muon candidates under consideration $\left(M\left(\mu^{+} \mu^{-}\right) \in[2,4] \mathrm{GeV}\right)$ an unbined maximum likelihood vertex fit is applied. The result is shown in Fig. 1.

The invariant mass resolution depends also on the pseudo-rapidity of the muon tracks. This is presented in Fig. 2, where the reconstructed $J / \psi$ candidates are shown in three regions of the pseudo-rapidity of the reconstructed muons:
- both in the barrel $(|\eta|<1.05)$,

- one in the barrel, one in the end-cap,

- both in the end-cap $(1.05<|\eta|<2.5)$.

Also the distributions for the prompt $J / \psi \mathrm{MC}$ and the results of the fit are shown as well. As expected, it was observed, that the invariant mass is $\sim 2.5$ times wider in the end-cap region as in the barrel region. This is due to the larger amount of material in the forward region. The good agreement with the MC expectations demonstrates, that the ATLAS detector is well described and understood already for the first data.

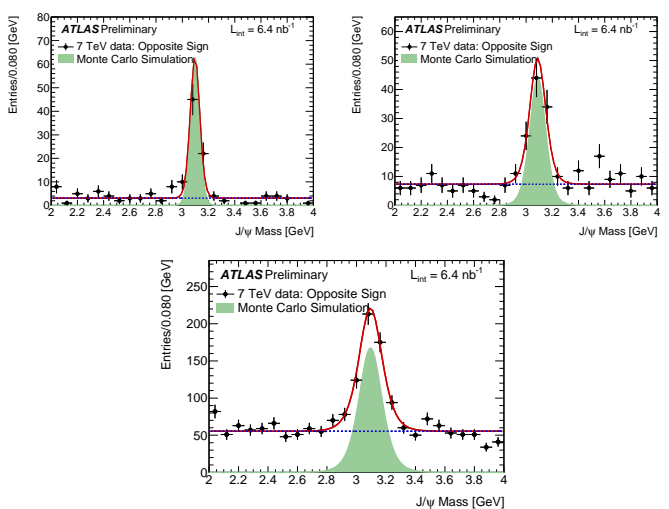

Figure 2. The invariant mass distribution for reconstructed $J / \psi \rightarrow \mu^{+} \mu^{-}$candidates in three different muon pseudo-rapidity regions: both muons in the barrel (top left), one muon in the end-cap, one in the barrel (top right) and both muons in the end-cap (bottom). The $\eta$ regions corresponding to barrel and end-cap are given in the text.

The analysis shown here has access to very low $p_{T}$ candidates of $J / \psi$ mesons producing soft $p_{T}$ muon tracks, which can nevertheless be reconstructed in the muon system reaching it mainly in the forward region where $p \gg p_{T}$. As a consequence, the $J / \psi$ candidates in this momentum regime have preferentially high rapidity. The 
kinematic properties of the event candidates in the $J / \psi$ mass region $\left(M_{J / \psi} \pm 3 \cdot \sigma_{M}\right)$ are shown in Fig. 3. It is possible to access the lowest possible $p_{T}(J / \psi)$ which in turn is a result of the muon acceptance of the ATLAS detector without any threshold requirement on the muon trigger. The shown distance between the two muons is given as $\Delta R=\sqrt{\Delta \eta^{2}+\Delta \phi^{2}}$. The MC distributions shown are normalized to the number of signal events found in data.
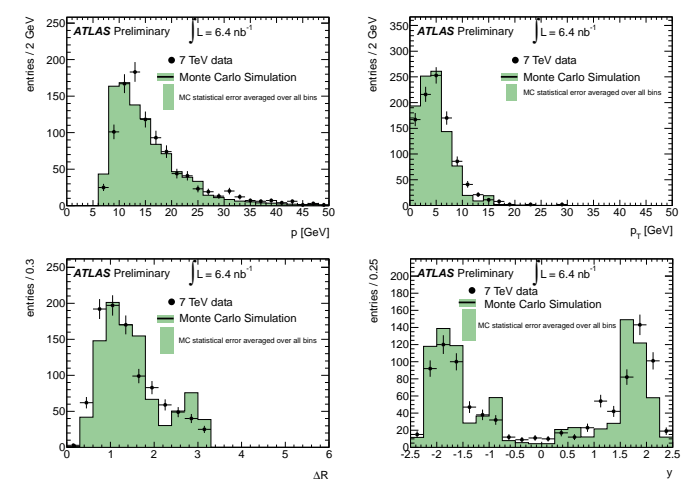

Figure 3. Kinematics of the $J / \psi \rightarrow \mu^{+} \mu^{-}$candidates: Momentum (top left), transverse momentum (top right), $\Delta R$ distance (bottom left) between two muons and rapidity distribution (bottom right) of the $J / \psi$ candidates in the mass region $M_{J / \psi} \pm 3 \sigma_{M}$. The black points correspond to data events in the signal region of the $J / \psi$, where the filled histograms are the expectations from the prompt $J / \psi$ and minimum bias MC.

\subsection{Open Charm}

The production of open charm mesons is one of the first hard QCD processes to be measured at LHC. Due to their large production cross sections and the clean decay signatures of $D$-mesons, a reasonable study of $D^{* \pm}, D^{ \pm}, D_{s}^{ \pm}$mesons is already possible with the first few data sets of ATLAS [1].

The data presented here, corresponding to an integrated luminosity of $1.4 \mathrm{nb}^{-1}$, were collected with the minimum-bias trigger only [7]. The open charm mesons reconstruction was done in the range of the transverse momentum $p_{T}\left(D^{(*)}\right)>$ $3.5 \mathrm{GeV}$ and the pseudo-rapidity of $\left|\eta\left(D^{(*)}\right)\right|<$ 2.1. The combinatorial background could significantly be reduced by the cuts $p_{T}\left(D^{* \pm}\right) / E_{T}>$ 0.02 and $p_{T}\left(D_{s}^{ \pm}\right) / E_{T}>0.04$, where $E_{T}$ is the sum of the transverse energy measured in the calorimeter and muon system. The charm meson candidates were reconstructed using tracks measured in the inner tracking detector [8] with an acceptance region of $\left|\eta_{\text {track }}\right|<2.5$.

The reconstruction of $D^{* \pm}$ mesons as shown in Fig. 4 is done via the well known decay channel $D^{* \pm} \rightarrow D^{0} \pi_{\mathrm{s}}^{ \pm} \rightarrow\left(K^{\mp} \pi^{ \pm}\right) \pi_{\mathrm{s}}^{ \pm}$. The $D^{ \pm}$mesons are reconstructed in the decay chain $D^{ \pm} \rightarrow K^{\mp} \pi^{ \pm} \pi^{ \pm}$. Due to the small mass difference $M\left(D^{* \pm}\right)-M\left(D^{0}\right)$, the "soft" pion $\pi_{\mathrm{s}}^{ \pm}$is constrained to have a limited momentum. Pairs of oppositely-charged tracks with $p_{T}>1.0 \mathrm{GeV}$ are combined to form $D^{0}$ candidates.

The reconstruction of $D_{s}^{ \pm}$mesons is done based on the decay $D_{s}^{ \pm} \rightarrow \Phi \pi^{ \pm}$with $\Phi \rightarrow K^{+} K^{-}$. The invariant mass $M(K \pi \pi)$ is shown in Fig. 5 for candidates with $\left|M(K K)-M_{\phi}\right| \leq 6 \mathrm{GeV}$ and clear signals can be found at the nominal values of $M\left(D_{s}^{ \pm}\right)$and $M(\Phi)$.

\subsection{Tagging of b-Quark Jets}

The presence of top-quarks are characterized by $b$ quarks in the final state, whereas the leading combinatorial backgrounds are composed of dominantly light quark jets. Consequently, one of the most important selection criteria for all analyzes and searches based on the reconstruction of events containing top quarks is the identification of $b$-quarks jets based on the specific properties of $b$-quark decays. The discrimination of $b$ - from light-quark jets originates mainly in the relatively long life time of $b$-flavored hadrons $(c \tau \approx 460 \mu \mathrm{m})$. This leads to measurable decay length $L$ for secondary vertices and transverse impact parameters $d_{0}$ of the decay products. The transverse impact parameter is defined as the transverse $(r / \phi)$-projection at the point of closest approach of the detached track to the primary vertex. Based on these discrimination variables $L$ and $d_{0}$, various $b$-tagging algorithms 

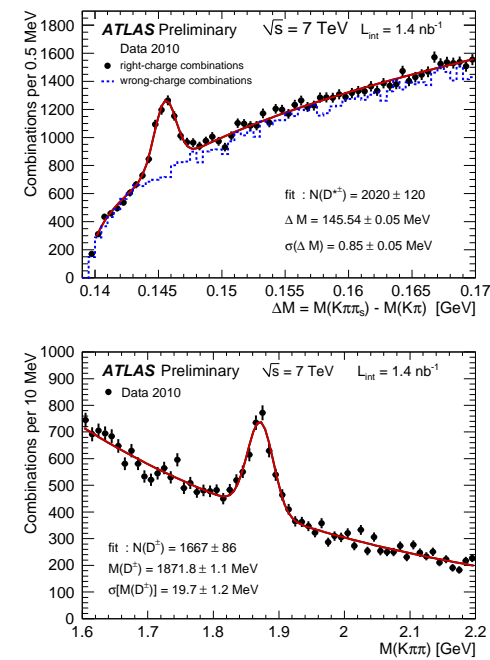

Figure 4. The distribution of the mass difference $\Delta M=M\left(K \pi \pi_{s}-M(K \pi)\right.$ (top) for the $D^{*}$ candidates. The dashed histogram shows the wrongcharge distribution and the solid line represents the fit result. The distribution of the invariant mass $M(K \pi \pi)$ for the $D^{ \pm}$candidates are shown in the histogram below.

can be defined providing $b$-weights reflecting the probability, that the selected jets originates from a $b$-quark. They are based on significances of the decay length $S_{L}=L / \sigma(L)$ or the impact parameters $S_{d_{0}}=d_{0} / \sigma\left(d_{0}\right)$, on resolution functions or on more complex ratios of probability density functions [1]. Furthermore, a good knowledge of impact parameters and secondary vertices is also crucial for many $B$-physics analyzes done at ATLAS.

With the first data collected with the ATLAS detector corresponding to and integrated luminosity of $\sim 1 \mathrm{nb}^{-1}$, the compatibility of the impact parameter [9] and decay length [10] significances and the corresponding MC expectations have been studied. Some results are presented in Fig. 6.
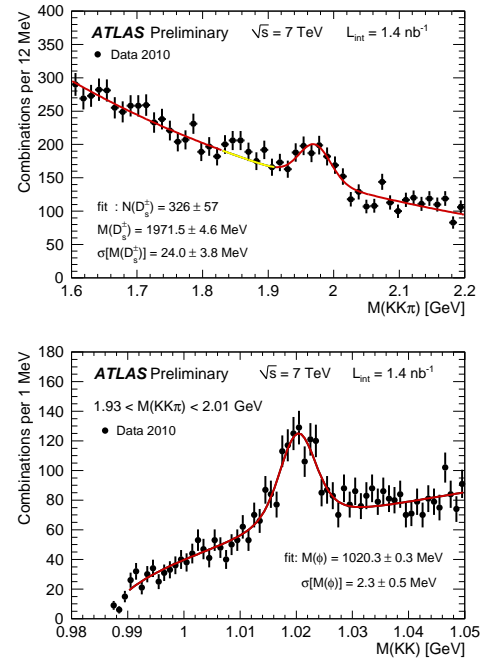

Figure 5. The $M(K K \pi)$ (top) and $M(K K)$ (bottom) distributions of the $D_{s}$-meson candidates.

\section{Long Term Perspectives}

The perspectives shown in this section are all based on simulations assuming a beam energy of $\sqrt{s}=14 \mathrm{TeV}$ reflecting the longer term goals of the ATLAS flavor physics program for more luminosity as has been published in the framework of the extended studies for the expected performance of the ATLAS experiment [1]. All this MC based studies have been done for a center of mass energy of $\sqrt{s}=14 \mathrm{TeV}$.

\subsection{Quarkonia}

One of the main prerequisites for successful quarkonia measurements is a reliable and well adjusted trigger strategy based on muon candidates. The low $p_{T}$ single- and di-muon triggers are suitable for control samples to be collected with the first beams at very low luminosities. The main trigger strategy is a di-muon trigger with a common vertex in the final state covering different mass ranges for the searches for $J / \psi$ - or $\Upsilon$ -mesons and the searches for very rare $B_{d, s} \rightarrow$ $\mu^{+} \mu^{-}$and the semi leptonic $B_{d, s} \rightarrow X_{s} \mu^{+} \mu^{-}$decays. The sidebands around the signal peaks are 

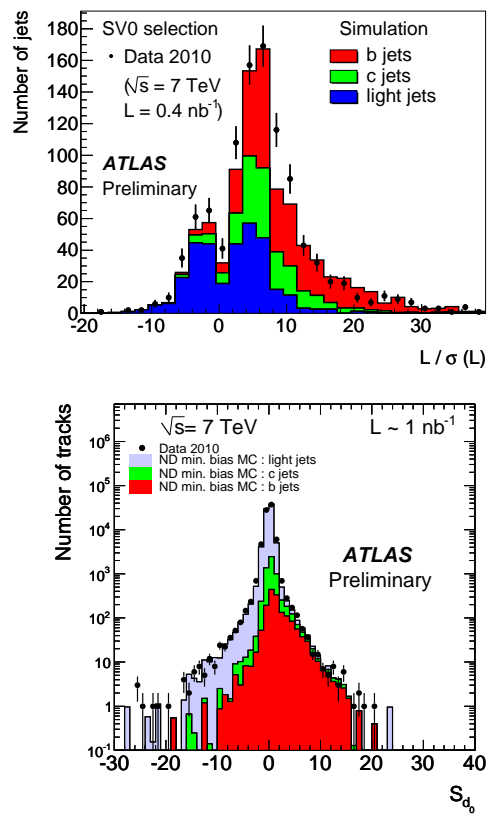

Figure 6. The distribution of the threedimensional decay length significance $S_{L}$ (top) and the second highest impact parameter significance $S_{d_{0}}$ (bottom) in the jet signed with respect to the calorimeter jet axis, for experimental data (black points) and for MC expectations (plain histograms) measured with non-diffractive minimum bias events. Both variables are used as $b$-jet discrimination weights within the first data.

needed to be covered to control the background contributions.

Quarkonia selection at ATLAS are mainly based on a di-muon trigger which requires two identified muons, both with $p_{T} \geq 4 / 6 \mathrm{GeV}$ and within a pseudo-rapidity of $|\eta|<2.4$. The dimuon sample considered here has offline $p_{T}$ cuts of 6 and $4 \mathrm{GeV}$ applied to the two identified muons. In Fig. 7 the resulting di-muon spectrum with background contributions is shown. To suppress backgrounds (decays-in-flight, heavy flavor decays) the tracks are required to come from the same vertex and with a pseudo-proper time cut

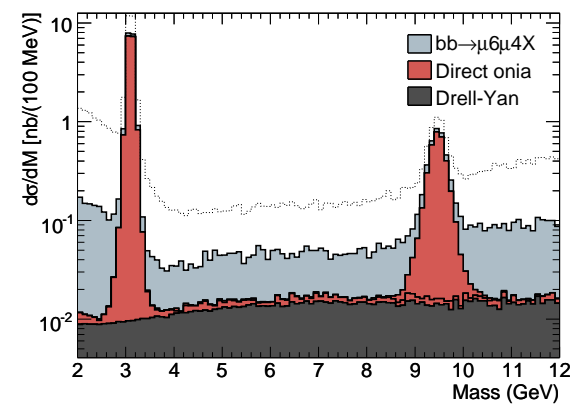

Figure 7. MC expectations of the invariant mass of di-muons from various sources, reconstructed with a di-muon trigger requiring $\left(p_{T}^{\mu} \geq 4 / 6 \mathrm{GeV}\right)$, that both muons are identified as coming from the primary vertex and a pseudo-proper time cut on $0.2 \mathrm{ps}$.

of $\tau=0.2 \mathrm{ps}$, defined as $\tau=\frac{M(J / \psi) \cdot L_{x y}}{p_{T}(J / \psi) \cdot c}$, where $L_{x y}$ is the transverse decay length of the di-muon. A further important measurement is the ratio $R_{p i}=\sigma(b \bar{b} \rightarrow J / \psi) / \sigma(p p \rightarrow J / \psi)$ of prompt and indirect $J / \psi$ production. With $\sim 1 \mathrm{pb}^{-1}$ of data, this ratio can be measured as a function of $p_{T}$ and $\eta$ at a precession of $\sim 10 \%$.

\section{2. $B^{+}$Meson}

The total $B^{+}$production cross section can be measured in the exclusive $B^{+} \rightarrow J / \psi K^{+}$decay channel with a reasonable statistical precision of $3 \%$ with the first $10 \mathrm{pb}^{-1}$ of data. It is an important reference channel for rare $B$-decays. The total and differential cross sections of rare decays can be be measured relative to the $B^{+}$production cross-section, thus allowing the cancellation of common systematic uncertainties [11].

The $B^{+}$invariant mass distribution $M\left(K^{+} \mu^{+} \mu^{-}\right)$of the candidates, fulfilling the selection cuts, is presented in Fig. 8 for signal and background with a maximum-likelihood fit, where the likelihood function is a Gaussian for the signal region and a linear function for the background $(b \bar{b} \rightarrow J / \psi+X)$. The mass range of the fit is taken from $5.15 \mathrm{GeV}$ to $5.8 \mathrm{GeV}$ 

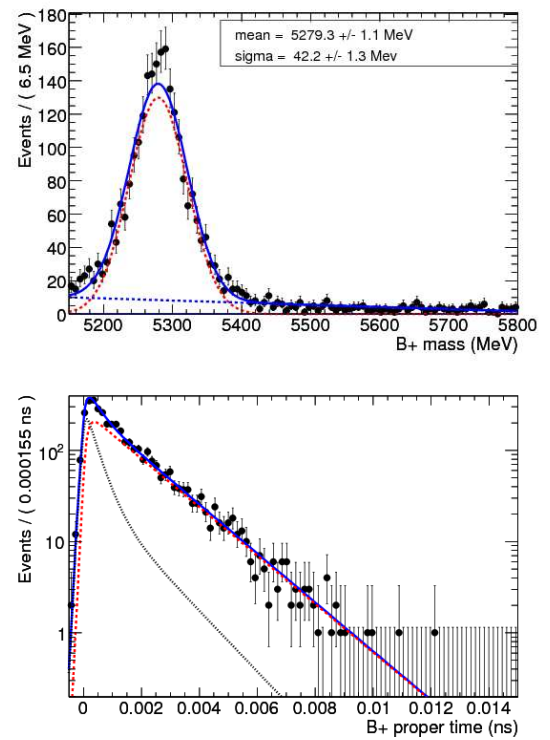

Figure $8 . B^{+}$mass fit with signal and background (top) and lifetime fit (bottom) with the signal (dashed red) and the background (dashed black) contributions shown separately.

in order to reduce contributions from partially reconstructed $B$ meson decays. The background at the right of the mass peak originates from misidentified $\pi^{+}$out of $B^{+} \rightarrow J / \psi \pi^{+}$decays. The relative statistical uncertainty, scaled properly for an integrated luminosity of $10 \mathrm{pb}^{-1}$, is $\sim 3 \%$. The slight shoulder to the left of the mass distribution is due to the background shape in this mass region and has been included in the systematic uncertainties of the fit model.

The measurement of the lifetime $\tau$ of the selected $B^{+}$candidates is a sensitive tool to confirm the beauty content in a sample, in particular the number of the reconstructed $B^{+} \rightarrow J / \psi K^{+}$ decays obtained in the $b \bar{b} \rightarrow J / \psi X$ data set. The proper decay-time distribution in the signal region $B^{+} \rightarrow J / \psi K^{+}$can be parametrized as a convolution of an exponential function with a Gaussian resolution function, while the background distribution parametrization consists of two dif- ferent exponential functions, where each is convoluted with a Gaussian resolution function. In the indirect production $b \bar{b} \rightarrow J / \psi X$ no zero lifetime events are expected since there is no prompt $J / \psi$ produced. In the realistic case, where zero lifetime events will be present, an extra Gaussian centered at zero is needed in order to properly describe those events. The results on the lifetime measurements are shown in Fig. 8.

\section{3. $B_{s} \rightarrow \mu^{+} \mu^{-}$Performance}

Flavor changing neutral currents, a direct transition from $b \rightarrow d / s$, are forbidden at the tree level in the SM and occur at the lowest order through one loop diagrams and are therefore highly suppressed $\left(\mathcal{B R}\left(B_{s} \rightarrow \mu^{+} \mu^{-}\right)_{\mathrm{SM}}=\right.$ $\left.(3.2 \pm 0.52) \cdot 10^{-9}\right)$. They are a sensitive test of the SM and its possible extension(s), providing information on the long distance QCD effects and enabling a determination of the CKM matrix elements $\left|V_{t d}\right|$ and $\left|V_{t s}\right|$. Furthermore, some of the rare decay channels contribute to the background for other channels, which are very sensitive to effects beyond the Standard Model. The so far largest $B_{s}^{0} \rightarrow \mu^{+} \mu^{-}$samples of $\mathcal{O}(10)$ candidates has been collected by the CDF collaboration within an integrated luminosity of $2 \mathrm{fb}^{-1}$ leading to the best upper limit for the branching ratio, which was recently published by the CDF collaboration. It was found to be $\mathcal{B R}\left(B_{s} \rightarrow\right.$ $\left.\mu^{+} \mu^{-}\right)_{\mathrm{CDF}}<5.8 \cdot 10^{-8}[12]$ and lies still on order of magnitude above the SM value.

Already at $1 \mathrm{fb}^{-1}$ ATLAS is able to collect $\mathcal{O}\left(10^{6}\right)$ di-muon events in the mass window of $4 \mathrm{GeV}<M\left(\mu^{+} \mu^{-}\right)<7 \mathrm{GeV}$. This is after the selection based on cuts on $p_{T}$, the invariant mass $M_{\mu^{+} \mu^{-}}$, the transverse decay length $L_{x y}$ of the di-muon system, and on isolation requirements. Based on this data, an upper limit on the number of signal events, $N_{B^{0}}$, corresponding to a given confidence level will be determined. The main challenge of this analyzes is to control the background sources originating from combinatorial decays as $b \bar{b} \rightarrow \mu^{+} \mu^{-} X$, from miss identifications $\left(B_{s}^{0} \rightarrow \pi^{+} \pi^{-}, B_{s}^{0} \rightarrow K^{+} K^{-}, B_{s}^{0} \rightarrow \pi^{+} K^{-} \nu_{\mu}\right)$ or from other rare decays $\left(B_{s}^{0} \rightarrow \mu^{+} \mu^{-} \mu^{+} \nu_{\mu}\right.$, $\left.B_{s}^{0} \rightarrow \mu^{+} \mu^{-} \gamma\right)$. Fig. 9 shows the di-muon mass distribution for signal and background events af- 


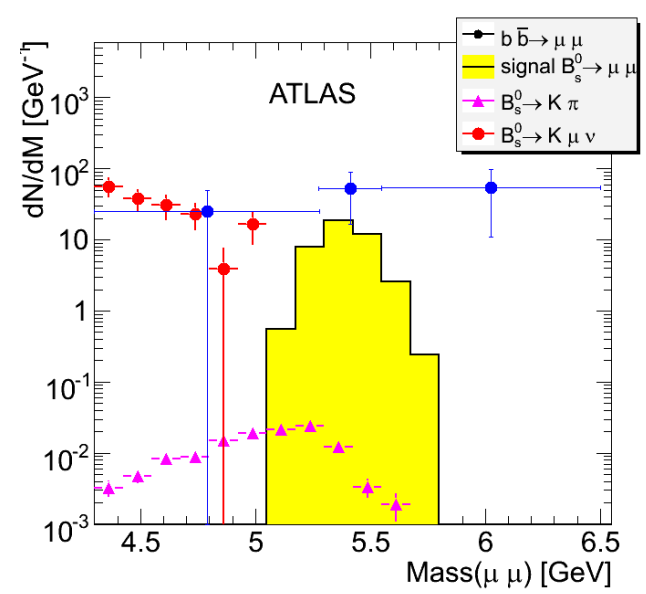

Figure 9. Di-muon mass distribution for surviving events after all selection cuts applied. The signal is shown as histogram, the combinatorial background as closed circles and the noncombinatorial background by open circles and triangles. The statistics corresponds to an integrated luminosity of $10 \mathrm{fb}^{-1}$.

ter all selection cuts have been applied. $N_{B^{0}}$ will be used to extract the upper limit on the $B_{s}^{0} \rightarrow \mu^{+} \mu^{-}$branching ratio $B R\left(B_{s}^{0} \rightarrow \mu^{+} \mu^{-}\right)$, using the reference channel $B^{+} \rightarrow J / \psi K^{+}$as described in Sec. 3.2, since trigger and offline reconstruction efficiencies largely cancel for di-muons in these channels. In this procedure, a ratio of geometric and kinematical acceptances of the signal and the reference channel will be determined from the MC simulations.

\section{Conclusions}

The first $J / \psi \rightarrow \mu^{+} \mu^{-}$resonances at ATLAS were observed with an integrated luminosity of $6.4 \mathrm{nb}^{-1}$ and the mass is in good agreement with the PDG value within the statistical uncertainty. The mass width is consistent with the MC expectation in all detector regions. Furthermore, clear $D^{* \pm}, D^{ \pm}$and $D_{s}^{ \pm}$signals could be reconstructed already within $1.4 \mathrm{pb}^{-1}$ of data. Also here, the values of the masses are in good agreement with the PDG world averages. This confirms nicely the high and well understood performance of the ATLAS detector for precision measurements.

Two examples of taggers for $b$-flavored jets are shown and found to be in good shape for data taking. The impact parameter and decay length significance of secondary vertices are in good agreement with the MC expectations. This is essential for the discrimination weights of the more sophisticated $b$-jet tagger, which are recently commissioned with the first data.

With respect to the long term prospective of the ATLAS flavor physics program, it could be shown, that flavor physics at ATLAS allows important measurements for the search for new physics. For this, a powerful and reliable di-muon trigger strategy is needed. Additionally, the flavor physics program provides valuable information on the detector performance already with the early results and supports the searches for new physics with calibration studies for $B$-physics.

\section{REFERENCES}

1. "The ATLAS Experiment at the CERN Large Hadron Collider", ATLAS Collaboration (G. Aad et al.), JINST 3:S08003,2008.

2. Particle Data Group (C. Amsler et al.), Phys.Lett.B667(2008-2009)no. 1-5,1-6.

3. T. Sjostrand, S. Mrenna, and P. Z. Skands, JHEP05(2006)026.

4. ATLAS Note ATL-PHYS-PUB-2010-002, CERN, March 2010.

5. A. Sherstnev and R. S. Thorne, Eur.Phys.J.C55(2008)553.

6. ATLAS Note ATLAS-CONF-2010-045, CERN, June 2010.

7. ATLAS Note ATLAS-CONF-2010-034, CERN, June 2010.

8. "Expected Performance of the ATLAS Experiment, Chapter 2: Tracking", ATLAS Collaboration, CERN-OPEN-2008-020 [arXiv:0901.0512].

9. ATLAS-CONF-2010-041, CERN, Mai 2010.

10. ATLAS-CONF-2010-042, CERN, Mai 2010.

11. W.-M. Yao et al., J. of Phys. G33(2006)1.

12. CDF Collaboration, PRL100(2008),101802 